\title{
Estado actual de la poliomielitis en Latinoamérica
}

\author{
Current status of poliomyelitis in Latin America
}

\author{
Luiza Helena Falleiros-Arlant ${ }^{1}$, Silvia E. González Ayala², Carla Domingues³, Jose Brea ${ }^{4}$ y Agustín De Colsa-Ranero 5
}

\author{
${ }^{1}$ Facultad de Medicina, Universidad Metropolitana de Santos. São Paulo, Brasil. \\ ${ }^{2}$ Facultad de Ciencias Médicas, Universidad Nacional La Plata. Buenos Aires, Argentina. \\ ${ }^{3}$ Doctora en Medicina Tropical por la Universidad de Brasilia, Brasil. \\ ${ }^{4}$ Sociedad Dominicana de Vacunologia. Santo Domingo, República Dominicana. \\ ${ }^{5}$ Instituto Nacional de Pediatría. Ciudad de México, México. \\ Información curricular de los autores al final del texto.
}

Una versión en inglés de este artículo se publica en este número de la revista.

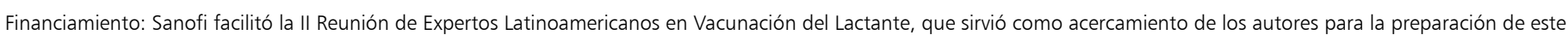
manuscrito. Sanofi no tuvo participación en el contenido ni en las conclusiones del manuscrito, que son responsabilidad exclusiva de los autores.

Recibido: 9 de noviembre de 2020

\section{Resumen}

La vacuna oral contra el poliovirus (OPV) ha sido fundamental en controlar la epidemia de poliomielitis, y destaca por su seguridad, eficacia, facilidad de administración oral y bajo costo. Sin embargo, a pesar de estas ventajas, al tratarse de una vacuna con virus vivos atenuados, existe la posibilidad de mutaciones que confieran neurovirulencia. Por ende, es importante la vigilancia de parálisis flácida aguda (PFA), ya sea asociada a las vacunas atenuadas (VAPP) o a los virus derivados de vacunas (VDPV). En esta revisión presentamos datos importantes de Latinoamérica en los últimos años, donde se revisan los datos de VDPV de transmisión comunitaria, de origen ambiguo y asociadas con inmunodeficiencias. Debido a la presencia de VDPV, es importante fortalecer el sistema de vigilancia epidemiológica por PFA, con datos muy inferiores a los recomendados en estos últimos años en las Américas. Adicionalmente, es fundamental mejorar las coberturas vacunales para reducir la cantidad de lactantes en riesgo de adquirir poliomielitis. En consecuencia, presentamos las tasas de cobertura vacunal con la vacuna inactivada contra el poliovirus (IPV) en la región y analizamos los programas de vacunación contra la poliomielitis en concordancia con las recomendaciones de la Sociedad Latinoamericana de Infectología Pediátrica (SLIPE; mínimo 3 dosis de IPV) y del Grupo de Expertos en Asesoramiento Estratégico (SAGE) sobre Inmunización de la OMS (mínimo 2 dosis de IPV). El estudio concluye con recomendaciones de los autores para el cambio de OPV a uso exclusivo de IPV, para aumentar las coberturas vacunales y para reforzar la vigilancia por PFA en la región.

Palabras clave: poliovirus; vacunación; Latinoamérica; recomendaciones.

\section{Abstract}

Oral poliovirus vaccine (OPV) has been instrumental in controlling the polio epidemic, and stands out for its safety, efficacy, ease of oral administration, and low cost. However, despite these advantages, as it is a live attenuated virus vaccine, there is the possibility of mutations that confer neurovirulence. Therefore, surveillance for acute flaccid paralysis (AFP) is important, whether associated with live vaccines (VAPP) or vaccine-derived viruses (VDPV). In this review we present important data from Latin America in recent years, where data on VDPV of community transmission, of ambiguous origin and associated with immunodeficiencies are reviewed. Due to the presence of VDPV, it is important to strengthen the epidemiological surveillance system for AFP, with data much lower than those recommended in recent years in the Americas. Additionally, it is essential to improve vaccination coverage to reduce the number of infants at risk of acquiring poliomyelitis. Consequently, we present the vaccination coverage rates with the inactivated vaccine against poliovirus (IPV) in the region and analyze the vaccination programs against poliomyelitis in accordance with the recommendations of the Latin American Society of Pediatric Infectious Diseases (SLIPE; minimum 3 doses of IPV) and the WHO Strategic Advisory Expert Group (SAGE) on Immunization (minimum 2 doses of IPV). The study concludes with recommendations from the authors for the change from OPV to exclusive use of IPV, to increase vaccination coverage and to strengthen surveillance for AFP in the region.

Keywords: poliovirus; vaccination; Latin America; recommendations. 


\section{Abreviaturas}

Se adopta nomenclatura internacional.

\begin{tabular}{|l|l|}
\hline aVDPV & poliovirus derivados de vacunas de origen ambiguo \\
\hline bOPV & OPV bivalente (serotipos 1 y 3) \\
\hline CoNaln & Comisión Nacional de Inmunizaciones (Argentina) \\
\hline COVID-19 & Enfermedad por coronavirus 2019 \\
\hline cVDPV & $\begin{array}{l}\text { Poliovirus derivados de vacunas circulante en la co- } \\
\text { munidad }\end{array}$ \\
\hline iVDPV & $\begin{array}{l}\text { Poliovirus derivados de vacunas en pacientes inmu- } \\
\text { nosuprimidos }\end{array}$ \\
\hline mOPV & OPV monovalente \\
\hline nOPV & Nueva formulación de OPV-2 \\
\hline OMS & Organización Mundial de la Salud \\
\hline OPS & Organización Panamericana de la Salud \\
\hline OPV & Vacuna oral contra poliovirus \\
\hline PFA & Parálisis flácida aguda \\
\hline SAGE & $\begin{array}{l}\text { Grupo de Expertos en Asesoramiento Estratégico sobre } \\
\text { Inmunización }\end{array}$ \\
\hline SARS-CoV-2 & $\begin{array}{l}\text { Coronavirus tipo } 2 \text { causante del síndrome respiratorio } \\
\text { agudo grave }\end{array}$ \\
\hline SLIPE & Sociedad Latinoamericana de Infectología Pediátrica \\
\hline tOPV & OPV trivalente \\
\hline UNICEF & Fondo de las Naciones Unidas para la Infancia \\
\hline VAPP & Poliomielitis paralítica asociada con la vacunación \\
\hline VDPV & Poliovirus derivados de vacunas \\
\hline wPV & Poliovirus salvaje \\
\hline
\end{tabular}

\section{Introducción}

$\mathrm{E}$ 1 virus de la poliomielitis es un enterovirus de la familia Picornaviridae. Existen tres tipos de poliovirus que difieren ligeramente en la proteína de la cápside $\mathrm{y}$, por ende, en su especificidad por los receptores y en su antigenicidad. El serotipo 1 en su forma salvaje (wPV-1) actualmente sólo se encuentra en dos países endémicos (Pakistán y Afganistán), mientras que los serotipos 2 y 3 en sus formas salvajes (wPV-2 y wPV-3, respectivamente) han sido erradicados. El último caso de wPV-2 se detectó en el año 1999 y fue declarado erradicado en el mundo en septiembre de 2015. Por su parte, el último caso de wPV-3 se detectó en el año 2012 y fue declarado erradicado en octubre de $2019^{1}$.

La historia de la poliomielitis sucedió en tres períodos bien definidos. El primer período fue endémico, ocurriendo hasta el siglo XIX, en el cual la infección por wPV determinaba la gravedad de la enfermedad. Posteriormente, el período epidémico ocurrió en el siglo XX con la urbanización, hasta la pandemia de 1955-1957, en la que los pulmotores (iron lungs) mejoraron las tasas de supervivencia, pero con secuelas graves. Con la distribución de la vacuna oral contra los poliovirus (OPV) en el año 1955 se inicia el período post-vacunal que logró erradicar dos de los tres serotipos de wPV.

En el año 1988, la Organización Mundial de la Salud (OMS) lanza la iniciativa para la erradicación de la poliomielitis. Tal iniciativa involucró campañas de vacunación masiva que lograron una importante reducción de la circulación de wPV. Al iniciar la campaña, se estimaban aproximadamente 350.000 casos de parálisis en más de 125 países, por los tres serotipos de wPV. Para el 22 de septiembre de 2020, la OMS ha comunicado 120 casos de $w P V-1^{2}$. A pesar de que no se ha logrado erradicar este serotipo, el progreso de la campaña sigue siendo notable, al lograr una reducción de casos por los tres serotipos de poliovirus superior a $99,9 \%$. Sin embargo, queda un largo y difícil camino para alcanzar la erradicación de la poliomielitis por wPV.

La Figura 1 presenta la evolución mundial de los casos de poliomielitis y los principales eventos que han ocurrido en el tiempo. Con el reconocido liderazgo del Dr. Ciro de Quadros ${ }^{3}$, el continente americano fue el primero en obtener la certificación de erradicación de los tres serotipos de wPV en el año 1994, después del último caso notificado en Perú en el año 1991. Otras regiones lograron eliminar la enfermedad más de una década después: Pacífico Occidental (2000), Europa (2002), Sudeste Asiático (2014) y, recientemente, África ${ }^{4}$ (2020). A la fecha, el wPV sólo es endémico en Afganistán y Pakistán, como ya se mencionó.

En abril de 2016, la OMS recomendó la transición mundial de la OPV trivalente (tOPV) a una vacuna bivalente (bOPV) que excluía el serotipo 2. Con esta estrategia se esperó reducir la circulación de poliovirus 2 derivado de vacunas (VDPV-2). Además, durante el mismo período se recomendó iniciar el esquema secuencial con al menos una dosis de IPV -preferiblemente la primera dosis-que ya se ha implementado en todos los países de la región de las Américas. 5 . Los calendarios vacunales difieren en cuanto a las fechas de administración e incorporan la IPV, ya sea en la serie primaria entre los 1 y 6 meses de edad además de un refuerzo, o bien, entre los 7 y 24 meses (p.ej.: Costa Rica) y entre los 4 y 7 años (ej., Uruguay).

A manera de ilustración, el último caso de poliomielitis por wPV en Argentina se registró en el año 1984. En respuesta a las recomendaciones de la $\mathrm{OMS}^{6}$, de la Sociedad Latinoamericana de Infectología Pediátrica (SLIPE) $)^{7,8} \mathrm{y}$ de expertos nacionales, la Comisión Nacional de Inmunizaciones (CoNaIn) de Argentina introdujo el plan de contención de poliovirus a través del uso de bOPV a partir del 2 de mayo de 2016 con uso de IPV a los 2 y 4 meses, en un esquema secuencial ${ }^{9}$. A través de la Resolución Ministerial 814/2020, se implementó el uso exclusivo de IPV en la serie primaria en el año 2020, con un refuerzo a los 5-6 años antes del ingreso escolar, a partir del 1 de junio de 2020 . 


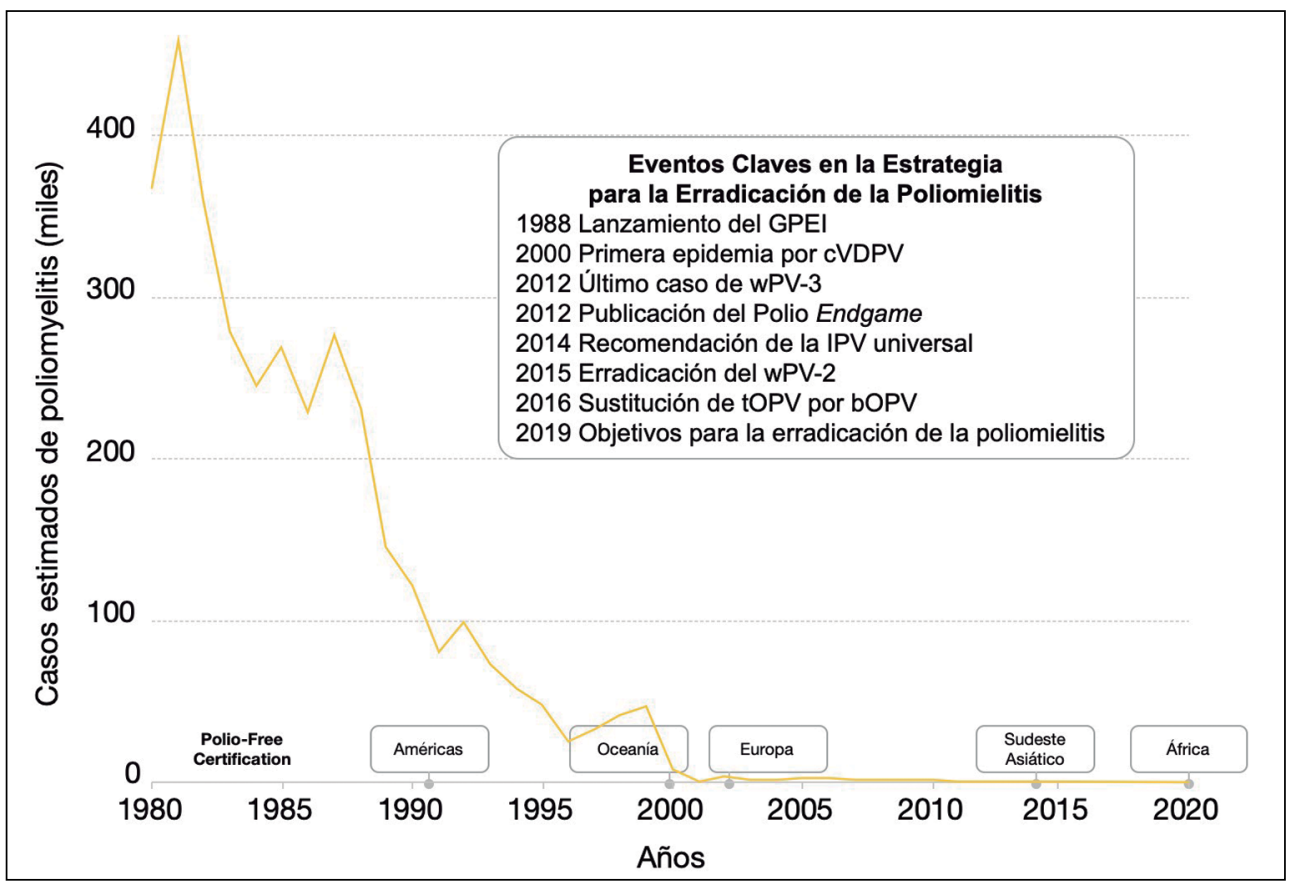

Figura 1. Evolución mundial de la frecuencia de casos de poliomielitis 1980-2020 y eventos claves en la estrategia de erradicación. Abreviaturas: cVDPV: poliovirus derivados de vacunas circulantes en la comunidad; GPEI: Estrategia Global para la Erradicación de Poliomielitis; bOPV: vacuna oral bivalente contra poliomielitis; OMS: Organización Mundial de la Salud; tOPV: vacuna oral trivalente contra poliomielitis; wP-2: poliovirus salvaje tipo 2; wP-3: poliovirus salvaje tipo 3. Fuente: preparado por los autores con datos de https://ourworldindata.org/polio y de la OMS (2020).

\section{Vacuna oral contra polio (OPV)}

La OPV contiene virus vivos atenuados y es segura y efectiva en la mayoría de los niños. Se estima que más de mil millones de niños han recibido la OPV y que esto ha evitado más de 650.000 casos de poliomielitis en el mundo desde el 2010. Sin duda, esta vacuna fue extremamente importante en el proceso de reducción mundial de la poliomielitis. Entre sus ventajas destacan la facilidad de su administración oral y el bajo costo, que contribuyeron con que la implementación fuera extremadamente práctica.

A pesar de su seguridad y eficacia, la OPV presenta algunas desventajas. Por ejemplo, para alcanzar una protección razonable, los lactantes necesitan de varias dosis. Además, al ser una vacuna de virus vivos atenuados, éstos son susceptibles de experimentar mutaciones y re-adquirir neurovirulencia. La poliomielitis de origen vacunal es indistinguible de la poliomielitis generada por wPV. Los virus vacunales también son excretados por vía fecal por más de dos meses y pueden infectar a otras personas en la comunidad.

Dependiendo de la intensidad de la divergencia genética, los poliovirus mutantes pueden provocar dos tipos de fenómenos de parálisis flácida aguda (PFA), a saber:

- Poliomielitis paralítica asociada con las vacunas atenuadas (VAPP).

- Poliomielitis paralítica por poliovirus derivados de vacunas (VDPV).
Las mutaciones y recombinaciones prácticamente están fuera de control de los sistemas de salud debido a que estos mecanismos son espontáneos en los virus circulantes ${ }^{10}$. Independiente del grado de divergencia genética, la VAPP y los VDPV pueden provocar poliomielitis con la misma clínica, intensidad y gravedad que los wPV. La única forma comprobada para evitar casos de VAPP y de VDPV es utilizar exclusivamente la IPV, que no contiene virus vivos y, por lo tanto, no tiene posibilidad de mutaciones.

\section{Poliomielitis paralítica asociada con las vacunas atenuadas (VAPP)}

Los casos de VAPP son raros, y al final de los años 80 , se reportaban uno caso de VAPP por cada 0,5 a 2,2 millones de dosis administradas. Los tres tipos de virus Sabin pueden causar VAPP y el riesgo es mayor después de la primera dosis de OPV y muy elevado en personas inmunocomprometidas. La Figura 2 presenta los casos de VAPP por número de dosis de OPV aplicadas. Datos de Brasil, donde el último caso de parálisis por wP fue identificado en el año 1989, muestran que en el período entre los años 2005 y 2014, se identificaron 94 casos de VAPP en menores de 5 años, ocasionados por los serotipos vacunales Sabin tipo 1 (19\%), tipo 2 (14\%), tipo 3 (31\%) y los aislamientos restantes de más de un serotipo $\operatorname{Sabin}^{11}$. 


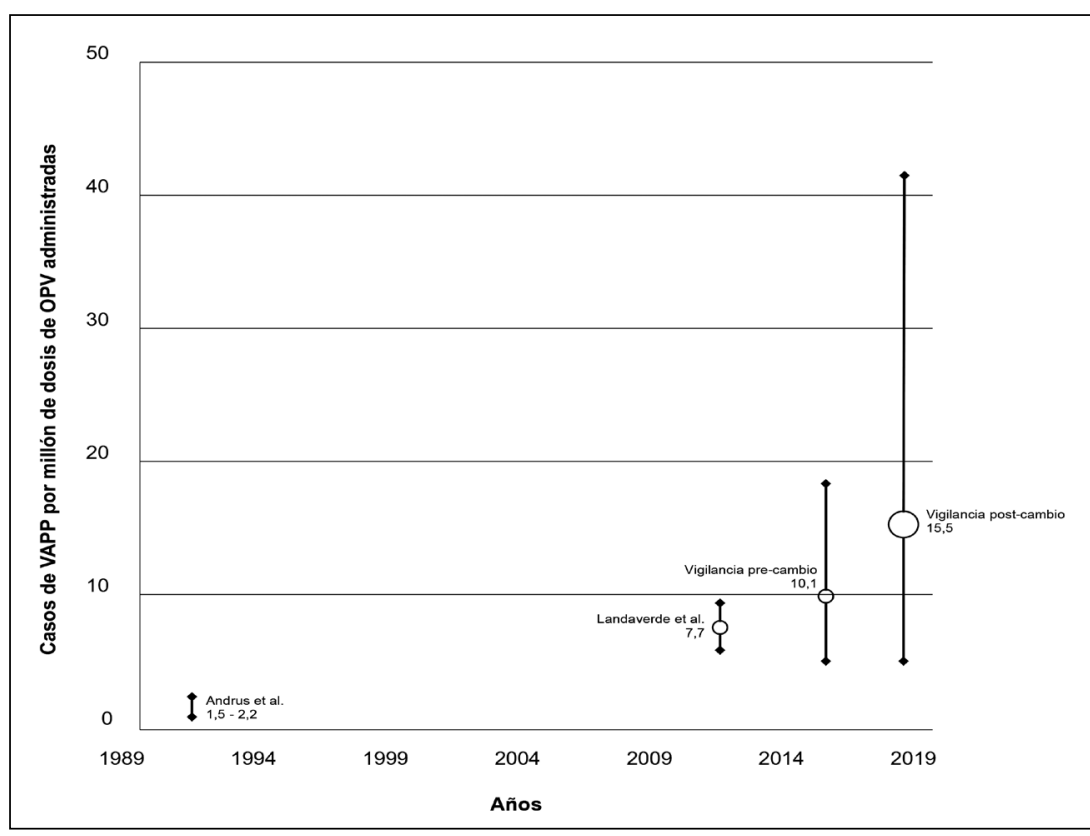

Figura 2. Tendencia de casos VAPP en la Subregión Latinoamericana y del Caribe, 1992-2018. bOPV: OPV bivalente (serotipos 1 y 3); OMS: Organización Mundial de la Salud; OPV: vacuna oral contra poliovirus; tOPV: OPV trivalente; VAPP: poliomielitis paralítica asociada con la vacunación. Fuente: Modificado por la autora (LHFA) con base en las referencias 12-14 y de los datos de vigilancia de la OMS antes y después del cambio de tOPV a bOPV.

\section{Parálisis flácida aguda por poliovirus derivados de vacunas (VDPV)}

Los VDPV afectan poblaciones no inmunizadas o sub-inmunizadas que viven en áreas con inadecuado saneamiento básico. Si la cobertura vacunal contra poliovirus permanece baja en la comunidad y el saneamiento es inadecuado (ej., no provisión de agua segura ni eliminación sanitaria de excretas), los VDPV aislados en el ambiente se pueden transmitir a poblaciones susceptibles, lo cual conlleva a emergencia de los tres tipos de VDPV.

\section{VDPV de circulación comunitaria (cVDPV)}

Los cVDPV se definen como aislamientos de VDPV para los que existe evidencia de transmisión de persona a persona en la comunidad. Los tres tipos de virus Sabin pueden experimentar mutaciones y causar parálisis; sin embargo, hasta el año 2016, la mayoría de los casos de cVDPV reportados eran por el serotipo 2 (cVDPV-2) ${ }^{15}$. Como ya se mencionó, en 2016 se propuso la transición de tOPV a bOPV. Dos hechos fundamentaron esta decisión: el wPV-2 ya había sido erradicado y el cVDPV-2 era el más frecuente. Con esta medida se esperaba reducir la circulación de VDPV. Además, el PV-2 interfiere con la respuesta inmunológica al PV-1 y al PV-3.

Con posterioridad al cambio a bOPV entre los años
2016 y 2018, se notificó un caso de VAPP por cada 15,5 millones de dosis administradas, pero se esperaba que esa reducción fuera más relevante, tal como había ocurrido en los Estados Unidos de América en la segunda mitad de los años 90. Por otra parte, se esperaba la eliminación total de los cVPDV-2. Por el contrario, se documentó un aumento dramático en brotes de cVDPV-2 en diversos países no endémicos. Al 29 de septiembre de 2020, se notificaron 409 casos por cVDPV-2 y 15 casos por cVDPV- $1^{16}$. A pesar de que actualmente sólo dos países tienen wPV endémico (Afganistán y Pakistán), más de 20 países no endémicos tuvieron brotes de cVDPV-2 y algunos también registraron brotes de cVDPV-1 durante el año 2020.

En el año 2018 se produjo un brote de 26 casos de cVDPV-1 en Papúa Nueva Guinea ${ }^{17}$, cuyo control costó más de 30 millones de dólares americanos (USD) y, en el año 2020, existe gran preocupación por un brote de cVPDV-1 en Yemen ${ }^{18}$. La mayoría de las epidemias con cVPDV-2 se reportan en África y afectan a niños que no han sido vacunados contra el serotipo 2. Paradójicamente, con la implementación de la vacuna monovalente (mOPV2), en un intento por controlar los brotes, el numero de brotes de cVPDV aumentó, tanto en países que implementaron la mOPV-2, como en aquéllos donde no se utilizó esta vacuna ${ }^{19}$.

A pesar de la exclusión del virus Sabin 2 de la tOPV, los casos de cVDPV-2 aumentaron de forma dramática: 2 durante el año 2016 y más de 500 hasta el 27 de octubre de $2020^{16}$. Por ello, se busca la aprobación en tiempo récord de una nueva vacuna oral (nOPV), más estable que los serotipos Sabin, y con baja probabilidad de revertir a neurovirulencia ${ }^{20}$. Se debe anotar que a pesar de que la probabilidad de reversión es baja, no se puede asegurar que será nula.

Los VDPV circulan en el ambiente en masa después de campañas de vacunación con la vacuna OPV-2, ya sea como componente de la tOPV o como mOPV-2. Por ejemplo, en México, se demostró que los cVDPV que circularon después de una campaña nacional de vacunación fueron aislados hasta los 8 meses siguientes ${ }^{21-23}$.

En Brasil, durante 3 años seguidos (2013-2015), la vigilancia ambiental realizada en algunos sitios de la ciudad de São Paulo reveló la presencia de virus Sabin tipo 1,2 y 3 después de las campañas nacionales de vacunación y antes de la introducción de la bOPV ${ }^{24}$. En Argentina ${ }^{25}$ y Brasil $^{26}$ se detectaron cepas Sabin en aguas ambientales, con mutaciones en la región genética asociada con neurovirulencia. Sin embargo, no hay reportes de casos de cVPDV en nuestra región.

\section{VDPV de origen ambiguo (aVDPV)}

Los aVDPV son aislamientos de VDPV en individuos o muestras ambientales, sin evidencia de circulación 
comunitaria o en individuos sin inmunodeficiencias documentadas $^{15}$. Hasta el 20 de octubre de 2020 se han documentado 268 aislamientos de aVDPV-2 y 8 aislamientos de aVDPV- $1^{16}$. La Tabla 1 presenta los casos de cVDPV y aVDPV reportados en el mundo entre el 01 de enero de 2016 y el 20 de octubre de 2020.

\section{VDPV en pacientes inmunosuprimidos (iVPDV)}

Los casos de iVDPV son muy inusuales, pero se estima que están sub-notificados, pues la mayoría fueron reportados en países de ingreso alto, con buenas condiciones para la vigilancia e investigación de casos. Además, no todos los casos de iVPDV se presentan con PFA, por lo que pueden escapar a los sistemas de vigilancia.

En un análisis de 107 casos compatibles con iVDPV al 30 de noviembre de 2016, 74 (70\%) se presentaron con VAPP. La edad media fue 1,04 años, lo cual implica que más de la mitad de los casos ocurrió en niños sobre 1 año de edad. La mayoría de los casos se registraron en países desarrollados y en $59 \%$ de los casos, la parálisis fue diagnosticada antes que la inmunodeficiencia primaria ${ }^{27}$.

Análisis posteriores de otras series de casos de iVDPV coincidieron en el comportamiento epidemiológico ${ }^{28,29}$. De 141 casos de iVDPV analizados, 58\% ocurrió en lactantes bajo un año de edad, $58 \%$ en varones, y casi la mitad fue reportada en países de ingreso medio-alto $(47,5 \%)$. En su mayoría se debieron a iVDPV-2 (58\%) e iVDPV-3 $(20 \%)^{28,29}$. Antes del cambio a IPV, 62\% de los casos se asociaba con iVDPV-2 (solo o asociado) y, a partir del año 2017, por iVDPV-1 e iVDPV- $3^{28}$.

En otra serie de 149 casos de iVDPV documentados entre los años 1961 y 2019, se encontró que $66 \%$ de los casos se diagnosticó en el período 2010-2019, 59\% en lactantes bajo 1 año de edad, $28 \%$ en 1-5 años, $13 \%$ en $>5$ años, $60 \%$ en varones, $64 \%$ con VAPP como síntoma de presentación y $56 \%$ causado por iVDPV-2, 23\% por iVDPV-3 y $17 \%$ por iVDPV $-1^{30}$. El estudio también destaca que $4 \%$ presentó combinaciones heterotípicas: iVDPV-2 con iVDPV-1 o con iVDPV-3 ${ }^{30}$. De los 149 casos de esta serie, 18 (12\%) casos se notificaron en las Américas. Es importante señalar la detección de cinco casos de iVDPV en Argentina por los tres serotipos: iVDPV-1 $(n=3)$, iVDPV-2 $(\mathrm{n}=1)$ y iVDPV-3 $(\mathrm{n}=1)^{12,13,31}$.

\section{Vigilancia de parálisis flácida aguda}

La vigilancia de PFA es la forma primaria para la detección de enfermedad por poliovirus. La calidad de tal vigilancia es fundamental para lograr la erradicación de la poliomielitis. Lamentablemente, se ha documentado un subregistro de casos en los sistemas de vigilancia en los países de la región, tanto en la notificación de PFA, como en la toma y envío de muestras en tiempo recomendado,
Tabla 1. Casos de cVPDV y de aVDPV (tipos 1,2, y 3 ) reportados en el mundo entre el 01 de enero de 2016 y el 20 de octubre de 2020

\begin{tabular}{cccccc}
\hline Virus & $\mathbf{2 0 1 6}$ & $\mathbf{2 0 1 7}$ & $\mathbf{2 0 1 8}$ & $\mathbf{2 0 1 9}$ & $\mathbf{2 0 2 0}^{\mathbf{a}}$ \\
cVDPV-1 & 3 & 0 & 27 & 12 & 17 \\
cVDPV-2 & 2 & 96 & 65 & 366 & 531 \\
cVDPV-3 & - & - & 7 & - & - \\
aVDPV-1 & - & - & 7 & 26 & 8 \\
aVDPV-2 & 5 & 2 & 65 & 196 & 268 \\
aVDPV-3 & - & - & 11 & - & -
\end{tabular}

aVDPV: poliovirus derivados de vacunas de origen ambiguo; cVDPV: poliovirus derivados de vacunas circulante en la comunidad. Fuente: Referencia 16 (ahasta la semana 20 octubre 2020).

con los formularios adecuadamente llenados y seguimiento de los casos, de acuerdo con las recomendaciones. La vigilancia de PFA se debe suplementar con la vigilancia ambiental, asegurando así que se cubren las fuentes de vigilancia de VDPV y potencialmente wPV importado. La mejoría en la sensibilidad de las actividades de vigilancia se basa en cinco pilares: detección efectiva, investigación, notificación, monitoreo y supervisión de los $\operatorname{casos}^{31}$.

Desde que se demostró que era posible erradicar la enfermedad, la notificación de PFA se mantiene por debajo de los casos esperados en la mayoría de los países de Latinoamérica. La Figura 3 presenta las tasas de notificación de casos de PFA en las Américas entre 2019 y 2020. Para este período se reportó un total de 1.424 casos de PFA $^{32}$.

Las tasas de notificación de PFA no alcanzan el mínimo recomendado ( 1 por 100.000 de habitantes $<15$ años) en la mayoría de los países del continente ${ }^{32}$. Sólo 4 países (México, Honduras, Costa Rica y Cuba; Figura 3, barras verdes) reportan tasas superiores a 1 , mientras que otros 6 países (Panamá, Colombia, El Salvador, Paraguay, Nicaragua, Venezuela; Figura 3, barras amarillas) se aproximan con tasas superiores a 0,80. Nueve países y la región del Caribe reportan tasas menores a 0,80 (Figura 3, barras rojas). De igual manera, no se logra el mínimo recomendado de muestras adecuadas durante las primeras 48 horas siguientes a la notificación ${ }^{32}$. De por sí esta situación es preocupante, y se agrava con la pandemia de la enfermedad producida por SARS-CoV-2, que ha suspendido y redirigido numerosos servicios esenciales de salud en Latinoamérica y el Caribe. Además, cuando se reabran las fronteras, cabe la posibilidad de re-introducción de wPV y/o cVDPV, especialmente con las coberturas vacunales sub-óptimas pre-pandémicas, que se han agravado con la pandemia.

Algunos estudios de vigilancia ambiental de poliovirus en las Américas muestran la efectividad del cambio de 
Figura 3. Tasas de notificación de parálisis flácida aguda en las Américas (por 100.000 habitantes menores de 15 años), según país, 2019-2020a. aDesde la semana epidemiológica 40 de 2019 hasta la semana epidemiológica 39 de 2020. Las abreviaturas corresponden al código ISO de cada país; CAR = Caribe; Estados Unidos no reporta datos al sistema de vigilancia; Ecuador y Uruguay no presentaron datos para el período del informe. Fuente: Referencia 32.

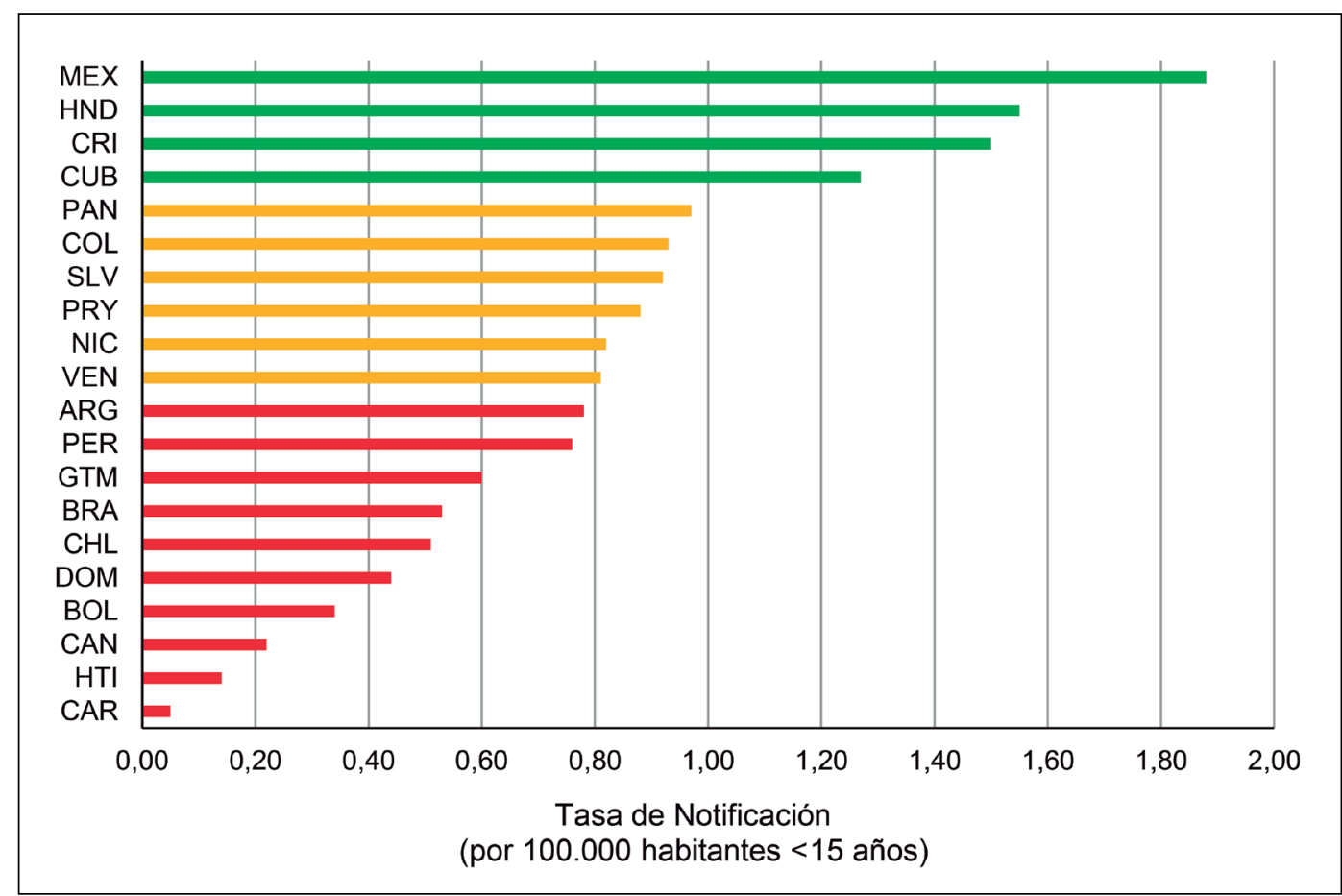

OPV a IPV para reducir la circulación de los poliovirus. Entre los años 2003 y 2005, la provincia de Córdoba, Argentina, implementó el cambio de OPV por IPV. Análisis al final del ciclo de implementación revelaron que los VDPV representaron 19\% de las muestras ambientales positivas. Al retornar al uso de OPV los siguientes dos meses, el porcentaje de VDPV en las muestras ambientales aumentó a $100 \%{ }^{25}$. Otro estudio de 31 muestras ambientales en Río de Janeiro, Brasil, entre los años 2011 y 2012 encontró enterovirus en 27 muestras, de los cuales 8 resultaron ser tipo Sabin (tipo $1=1$; tipo $2=5$; tipo $3=2$ ), sin evidencia de VDPV ni de recombinaciones ${ }^{26}$. Más recientemente, entre los años 2016 y 2017 en Puerto Príncipe y Gonaïves, Haití, se encontraron enterovirus no polio y poliovirus Sabin tipo 1 y 2, pero no se encontraron cepas de VPDV ni de wPV ${ }^{33}$.

\section{Coberturas vacunales en América Latina}

Según la OPS, los países latinoamericanos presentan coberturas vacunales demasiado bajas y heterogéneasentre 81 y $95 \%$-lo cual no permite lograr la eficacia vacunal ${ }^{14}$. En la práctica, estas cifras resultan en grandes poblaciones con riesgo de un re-brote de poliomielitis. Por ejemplo, Ecuador reporta aproximadamente $70 \%$ de sus niños no vacunados con la primera dosis de IPV, que pone a más de 100.000 niños en riesgo de wPV- $2^{35}$. Utilizando datos de 2019, la UNICEF ${ }^{35}$ estimó que Venezuela, con $55 \%$ de cobertura con una dosis de IPV, tiene 226.000 niños en riesgo de poliomielitis. El mismo año, las coberturas con una dosis de IPV de Brasil y México fueron relativamente más altas ( 86 y 84\%, respectivamente) pero, por la densidad de población de estos países, se estima que el total de niños en riesgo de poliomielitis es de 400.000 en Brasil y 348.000 en México $^{35}$.

\section{La Estrategia Final (Endgame) 2019-2023 para la erradicación de la poliomielitis}

La Estrategia Final se basa en tres objetivos durante el lustro 2019- $2023^{15}$ :

- Interrumpir la circulación de wPV.

- Interrumpir la transmisión de los VDPV dentro de los 120 días de la detección de un brote y eliminar el riesgo de una futura reemergencia y

- Lograr y mantener coberturas vacunales superiores a $95 \%$.

Aunque la OPV siga siendo extremadamente útil en el proceso de eliminación de la poliomielitis en las Américas, es la principal causa de circulación silenciosa de VDPVs ${ }^{20}$. Hasta que se disponga de una vacuna sin riesgo de reversión, la Estrategia Final ${ }^{15}$ implica el uso exclusivo de tres o más dosis IPV con una alta cobertura 


\begin{tabular}{|c|c|c|}
\hline Dosis de IPV & Países & Esquema \\
\hline 5 & Costa Rica & $\begin{array}{l}\text { Vacuna pentavalente aP-IPV a los 2, 4, } 6 \text { y } 18 \text { meses y refuerzo con Tdap-IPV en preescolares. } \\
\text { No utiliza OPV }\end{array}$ \\
\hline \multirow[t]{3}{*}{4} & Argentina y Uruguay & IPV sola a los 2, 4 y 6 meses y refuerzo a los $4-6$ años. No utilizan OPV \\
\hline & Chile & Vacuna hexavalente aP-IPV a los 2, 4 y 6 meses y refuerzo a los 18 meses. No utiliza OPV \\
\hline & México & $\begin{array}{l}\text { Vacuna hexavalente aP-IPV a los } 2,4 \text { y } 6 \text { meses y refuerzo a los } 18 \text { meses. Aun recomienda } \\
\text { refuerzo con bOPV en preescolares y durante campañas de vacunación }\end{array}$ \\
\hline \multirow[t]{2}{*}{3} & Brasil y Colombia & $\begin{array}{l}\text { IPV sola a los } 2,4 \text { y } 6 \text { meses y refuerzos con bOPV a los } 18 \text { meses y entre los 4-6 años o } \\
\text { durante campañas de vacunación }\end{array}$ \\
\hline & Panamá & Vacuna hexavalente aP-IPV a los 2,4 y 6 meses y bOPV a los 18 meses y entre los 4 y 6 años \\
\hline \multirow[t]{3}{*}{2} & El Salvador, Guatemala, Honduras, Paraguay y Perú & 2 dosis de IPV sola a los 2 y 4 meses; $3^{a}$ dosis y refuerzos con bOPV \\
\hline & Ecuador & 2 dosis fraccionadas de IPV, otras dosis con bOPV \\
\hline & Cuba & 2 dosis fraccionada de IPV a los 4 y 8 meses, otras dosis con bOPV \\
\hline 1 & Bolivia, Haití, Nicaragua, República Dominicana, Venezuela & IPV sola a los 2 meses; $2^{a}$ y $3^{a}$ dosis con bOPV, otras dosis con bOPV \\
\hline
\end{tabular}

aP: vacuna acelular contra la tos ferina; bOPV: OPV bivalente; IPV: vacuna inactivada contra la poliomielitis; OPV: vacuna oral contra la poliomielitis; Tdap: vacuna contra el tétano, la difteria y aP. Fuente: Modificado de referencia 36.

vacunal $(\geq 95 \%)^{34}$. Es por ello que la SLIPE presentó una recomendación de cuatro dosis de IPV (3 en la serie primaria +1 refuerzo), ya sea monovalente o polivalente en combinación con otras vacunas del programa de inmunizaciones de cada país de la región ${ }^{7}$.

A la fecha, todos los países latinoamericanos han introducido como mínimo una dosis de IPV (Tabla 2). Cinco países (Costa Rica, México, Chile, Uruguay y Argentina) han implementado la recomendación de SLIPE de al menos 4 dosis de IPV. A excepción de México, el resto de estos países ha dejado de utilizar la OPV por completo.

En octubre de 2020, el Grupo de Expertos en Asesoramiento Estratégico (SAGE) sobre Inmunización de la OMS recomendó que todos los países del mundo que utilizaban una dosis de IPV pasen a utilizar dos dosis de IPV para aumentar la protección contra el wPV-2 ${ }^{37}$. En América Latina, esta recomendación afecta los calendarios vacunales de Bolivia, Haití, Nicaragua, República Dominicana y Venezuela (Tabla 2).

\section{Conclusiones y Recomendaciones}

Tres elementos claves en América Latina aumentan significativamente el riesgo de brotes de poliomielitis: el uso continuado de la OPV, las bajas coberturas vacunales y la vigilancia ineficiente de PFA. Por lo tanto:
- Mientras se utilice la OPV disponible hoy, continuaremos observando la presencia de VDPV, ya que el virus seguirá circulando y puede provocar poliomielitis en la población susceptible, especialmente en niños. Con los datos que se dispone hasta ahora, sólo se erradicará la poliomielitis, discontinuando la OPV y utilizando solamente la IPV.

- Mantener un esquema de no menos de 3 dosis de IPV, retirando del esquema la OPV. Se recomienda incorporar una cuarta dosis a los 18 meses y, de ser posible, una quinta dosis entre los 4 y 6 años para mantener la inmunidad durante más tiempo.

- En los años previos, las coberturas vacunales ya se mostraban bajas, especialmente en el año 2019. Tal situación se agravó con las medidas restrictivas impuestas por la COVID-19, disminuyendo aún más las coberturas y dejando millares de niños desprotegidos a nivel global, incluyendo los niños latinoamericanos.

- Es fundamental mejorar la vigilancia de PFA para la detección de casos de wPV, VAPP, cVDPV, iVDPV y de casos asociados a la bOPV. Es esencial tener una vigilancia epidemiológica de la PFA de alta calidad con vigilancia ambiental. A pesar de ser costosa y de difícil realización, la vigilancia ambiental es fundamental para suplementar la vigilancia epidemiológica de PFA.

El control y la mejoría de estos tres elementos claves en América Latina siguen siendo prioritarios en el proceso 
de erradicación de la poliomielitis. Los gobiernos deben hacer todo lo posible para asegurar el funcionamiento adecuado de estos pilares, que incluye:

- Prioridad en la vacunación, con acciones complementarias y sostenibles de vacunación, tanto intra como extra-muros, hasta lograr una cobertura igual o mayor a $95 \%$.

- Asignación de recursos para la vacunación.

- Política sostenida para la asignación de recursos y priorización de la vacunación.

- Estrategia de comunicación para dar credibilidad y confianza en el proceso de vacunación.

- Liderazgo nacional y confianza pública.

Agradecimientos. Los autores desean reconocer la colaboración de Humberto López Castillo, MD, PhD, CPH, CMI en la preparación y revisión del manuscrito, incluyendo las tablas y figuras.

Luiza Helena Falleiros-Arlant, MD

- Departamento de Salud de la Niñez, Facultad de Medicina, Universidad Metropolitana de Santos. São Paulo, Brasil

- Departamento de Infectología (Sociedad Brasileña de Pediatría) y Comité de Infectología, Sociedad de Pediatría de São Paulo, Brasil

- Comité Científico y Comité de Vacunas, Sociedad Latinoamericana de Infectología Pediátrica (SLIPE).

- Ex presidente (2013-2015) y actual miembro del Núcleo Asesor Permanente de la SLIPE.
Silvia E. González Ayala, MD

- Facultad de Ciencias Médicas, Universidad Nacional La Plata. Buenos Aires, Argentina.

- Comité Institucional de Revisión de Protocolos de Investigación, Hospital de Niños Sor María Ludovica. Buenos Aires, Argentina.

- Comisión Nacional de Seguridad en Vacunas (Co$\mathrm{NaSeVa}$ ), Ministerio de Salud de la Nación. Buenos Aires, Argentina

Carla Domingues, PhD, MPH

- Especialista en Epidemiología por la Universidad de São Paulo/Brasil, Universidad del Sur de la Florida/ USA y por la Universidad Johns Hopkins/USA.

- Doctora en Medicina Tropical por la Universidad de Brasilia/Brasil.

- Universidade de Brasília. Brasília, Brasil.

José Brea Del Castillo, MD

- Sociedad Dominicana de Vacunologia. Santo Domingo, República Dominicana.

- Comité Científico, Sociedad Dominicana de Pediatría. Santo Domingo, República Dominicana.

- Comité de Vacunas de la Sociedad Latinoamericana de Infectología Pediátrica (SLIPE).

Agustín De Colsa-Ranero, MD

- Laboratorio de Microbiología Molecular y Departamento de Enfermedades Infecciosas Pediátricas, Instituto Nacional de Pediatría. Ciudad de México, México.

\section{Referencias bibliográficas}

1.- WHO. Two out of three wild poliovirus strains eradicated. https://www.who.int/news- room/ feature-stories/detail/two-out-of-three-wildpoliovirus-strains-eradicated

2.- WHO. Global Wild Poliovirus 2015-2020. 2020. http://polioeradication.org/wp- content/ uploads/2020/09/weekly-polio-analysesWPV-20200922.pdf

3.- Ciro de Quadros, pioneer of polio eradication, is honored as a PAHO Public Health Hero of the Americas. PAHO. https://www.paho.org/hq/ index.php?option $=$ com_content\&view $=$ article \&id=9493:2014- ciro-quadros-pioneer-polioeradication-honored-paho-public-health-heroamericas\&Itemid $=135 \&$ lang $=\mathrm{en}$

4.- WHO. Global polio eradication initiative applauds WHO African region for wild polio-free certification. https://www.who.int/ news-room/detail/25-08-2020-global-polio- eradication- initiative-applauds-who-africanregion-for-wild-polio-free-certification

5.- Hampton L M, Farrell M, Ramírez-González A, Menning L, Shendale S, Lewis I, et al. Cessation of trivalent oral poliovirus vaccine and introduction of inactivated poliovirus vaccine - worldwide, 2016. MMWR Morb Mortal Wkly Rep. 2016; 65 (35): 934-8. doi: 10.15585/mmwr.mm6535a3.

6.- WHO, Polio Global Eradication Initiative. Polio Endgame Strategy 2019-2023: Eradication, integration, certification and containment. 2019. http://polioeradication.org/ wp- content/uploads/2019/06/english-polioendgame-strategy.pdf

7.- Falleiros-Arlant L H, Avila-Aguero M L, Brea del Castillo J, Marino C. The challenge of changing the inactivated poliomyelitis vaccine in Latin America: declaration of the Latin American Society of Pediatric Infectious Diseases (SLIPE). Rev Chilena Infectol.
2014; 31(5): 590-603. doi: 10.4067/S071610182014000500012.

8.- Arbo A, Falleiros-Arlant L H, López EL, Brea Del Castillo J, Martínez De Cuellar C, Moreno G, et al. [Remarks on the possibility of the introduction of fractionated dose of the inactivated poliomyelitis vaccine in the Latin American Child Immunization Schedule]. Rev Chilena Infectol 2018; 35 (4): 395-402. Consideraciones referente a la posibilidad de introducir la dosis fraccionada de la vacuna antipoliomielitis inactivada en el Calendario de Inmunizaciones del Niño Latinoamericano. doi:10.4067/s0716-10182018000400395.

9.- Rüttimann R, Contrini M M, Rearte A, González S, Pujadas M, Torres J P, et al. Documento de posición intersocietario: Actualidad en poliomielitis: Recomendaciones sobre vacunación en Argentina. 2019. https:// drive.google.com/file/d/1xhQB8vYR8vQbK82QbrrJGhMzmQRoB6O/view 
10.- Muslin C, Mac Kain A, Bessaud M, Blondel B, Delpeyroux F. Recombination in Enteroviruses, a multi-step modular evolutionary process. Viruses 2019;11 (9). doi: 10.3390/v11090859.

11.- Sousa I P, Jr, Burlandy F M, Oliveira S S, Nunes A M, Sousa C, da Silva E M. Acute flaccid paralysis laboratorial surveillance in a polio-free country: Brazil, 2005-2014. Hum Vaccin Immunother. 2017; 13 (3): 717-23. doi: 10.1080/21645515.2016.1236164.

12.- Andrus J K, de Quadros C, Olive J M, Hull H F. Screening of cases of acute flaccid paralysis for poliomyelitis eradication: ways to improve specificity. Bull World Health Organ. 1992; 70(5): 591-6. PMID: 1281445.

13.- Landaverde J M, Trumbo S P, DanovaroHolliday M C, Cochi S E, Gandhi R, RuizMatus C. Vaccine-associated paralytic poliomyelitis in the postelimination era in Latin America and the Caribbean, 1992-2011. J Infect Dis. 2014; 209 (9): 1393-402. doi:10.1093/ infdis/jit602.

14.- WHO. Regional Summary - Region of the Americas. Accessed 02 October 2020, https:// extranet.who.int/polis/public/CaseCount.aspx

15.- Polio Global Eradication Initiative. Classification and reporting of vaccine derived poliovirus (VDPV): GPEI guidelines. 2016. http://polioeradication.org/wp- content/ uploads/2016/09/Reporting-and-Classificationof-VDPVs_Aug2016_EN.pdf.

16.- WHO. Global circulating vaccine-derived poliovirus (cVDPV). 29 September 2020. http://polioeradication.org/wp-content/ uploads/2020/10/weekly-polio-analysescVDPV- 20200929.pdf.

17.- WHO. 18th Meeting of the SAGE Polio Working Group. Conclusions and recommendations: note for the record 2019. https://www.who.int/immunization/sage/ meetings/2019/october/1_18th_Meeting_SAGE_WG_on_Polio_-_Note_for_Record.pdf.

18.- Global Polio Eradication Initiative. Polio this week in Yemen. 02 October 2020, http:// polioeradication.org/where-we-work/poliooutbreak-countries/yemen/

19.- Jorba J, Diop O M, Iber J, Henderson E, Zhao $\mathrm{K}$, Quddus A, et al. Update on vaccine-derived poliovirus outbreaks - worldwide, January 2018-June 2019. MMWR Morb Mortal Wkly Rep. 2019; 68 (45): 1024-8. doi:10.15585/ mmwr.mm6845a4.
20.- Roberts L. Global polio eradication falters in the final stretch. Science. 2020; 367(6473): 1415. doi: 10.1126/science.367.6473.14.

21.- Troy S B, Ferreyra-Reyes L, Huang C, Sarnquist C, Canizales-Quintero S, Nelson C, et al. Community circulation patterns of oral polio vaccine serotypes 1, 2, and 3 after Mexican national immunization weeks. J Infect Dis 2014; 209 (11): 1693-9. doi: 10.1093/infdis/ jit831

22.- Estivariz C F, Pérez-Sánchez E E, Bahena A, Burns C C, Gary Jr H E, García-Lozano H, et al. Field performance of two methods for detection of poliovirus in wastewater samples, Mexico 2016-2017. Food Environ Virol. 2019; 11(4): 364-73. doi:10.1007/s12560-019-093999.

23.- Altamirano J, Purington N, Behl R, Sarnquist C, Holubar M, García-García L, et al. Characterization of household and community shedding and transmission of oral polio vaccine in Mexican communities with varying vaccination coverage. Clin Infect Dis 2018; 67 (Suppl_1): S4-S17. doi:10.1093/cid/ciy650.

24.- Ministério da Saúde do Brasil. Monitoramento Ambiental do Polio. http://www.saude.sp.gov. $\mathrm{br} /$ resources/ccd/noticias/monitoramento ambiental do polio - cetesb.pdf

25.- Mueller JE, Bessaud $\bar{M}$, Huang QS, Martínez LC, Barril PA, Morel V, et al. Environmental poliovirus surveillance during oral poliovirus vaccine and inactivated poliovirus vaccine use in Cordoba Province, Argentina. Appl Environ Microbiol. 2009;75 (5): 1395-401. doi:10.1128/ AEM.02201-08.

26.- de Oliveira Pereira J S, da Silva L R, de Meireles Nunes A, de Souza Oliveira S, da Costa E V, da Silva E E. Environmental surveillance of polioviruses in Rio de Janeiro, Brazil, in support to the activities of Global Polio Eradication Initiative. Food Environ Virol. 2016; 8(1): 27- 33. doi:10.1007/s12560015-9221-5

27.- Shaghaghi M, Soleyman-Jahi S, Abolhassani H, Yazdani R, Azizi G, Rezaei N, et al. New insights into physiopathology of immunodeficiency-associated vaccine-derived poliovirus infection; systematic review of over 5 decades of data. Vaccine. 2018;36 (13): 1711 9. doi: 10.1016/j.vaccine.2018.02.059.

28.- WHO. Poliovirus surveillance among patients with primary immunodeficiency disorders (PIDs): Introduction of new guidelines for the
SAGE meeting. 2019. https://www.who.int/ immunization/sage/meetings/2019/april/2_ SAGE April 2019 polio_Mach.pdf.

29.- Macklin G, Liao Y, Takane M, Dooling K, Gilmour S, Mach O, et al. Prolonged excretion of poliovirus among individuals with primary immunodeficiency disorder: an analysis of the World Health Organization registry. Front Immunol. 2017; 8: 1103. doi:10.3389/ fimmu.2017.01103.

30.- Macklin G, Diop O M, Humayun A, Shahmahmoodi, S, El-Sayed ZA, Triki H, et al. Update on immunodeficiency-associated vaccine-derived polioviruses - worldwide, July 2018-December 2019. MMWR Morb Mortal Wkly Rep 2020; 69 (28): 913-7. doi:10.15585/ mmwr.mm6928a4

31.- Lickness J S, Gardner T, Diop O M, Chavan S, Jorba J, Ahmed J, et al. Surveillance to track progress toward polio eradication - worldwide, 2018-2019. MMWR Morb Mortal Wkly Rep. 2020; 69 (20): 623-9. doi:10.15585/mmwr. $\mathrm{mm} 6920 \mathrm{a} 3$.

32.- PAHO. Acute flaccid paralysis surveillance in the Americas. Polio Weekly Bulletin 2020; 35 (14): $1-2$.

33.- Coulliette-Salmond A D, Alleman M M, Wilnique P, Rey-Benito G, Belgasmi Wright $\mathrm{H}$, Wielgus Hecker J, et al. Haiti poliovirus environmental surveillance. Am J Trop Med Hyg. 2019; 101 (6): 1240-8. doi: 10.4269/ ajtmh.19-0469.

34.- Alfaro-Murillo J A, Avila-Aguero M L, Fitzpatrick M C, Crystal C J, Falleiros-Arlant L H, Galvani A P. The case for replacing live oral polio vaccine with inactivated vaccine in the Americas. Lancet. 2020;395(10230):11631166. doi:10.1016/S0140-6736(20)30213-0.

35.- UNICEF. Estimated immunization coverage and number of unvaccinated children, IPV1, 2019. Retrieved from: https://data.unicef.org/ resources/immunization-coverage-estimatesdata-visualization/

36.- WHO. Immunization Monitoring Global Summary. Retrieved from: https://apps.who.int/ immunization_monitoring/globalsummary/coun tries?countrycriteria $\% 5$ Bcountry.

37.- WHO. Highlights from the Meeting of the Strategic Advisory Group of Experts (SAGE) on immunization. Retrieved from: https://www. who.int/docs/default-source/immunization/ sage/2020/october/highlights-sage-october2020-meeting-final 